\title{
Analysis of BIM Technology to Optimize the Effect and Economic Benefit in Project Management
}

\author{
Shuguo Zhang ${ }^{\mathrm{a}}$, Hong $\mathrm{Xu}^{\mathrm{b} \text {, * }}$ \\ ${ }^{1}$ Department of Economics and Management, North China Electric Power University, Baoding. \\ 071003, China \\ aemail: 13930865901@163.com, bemail: xuhong_880925@126.com, "corresponding author
}

Keywords: BIM technology, project management, optimization function, economic performance

\begin{abstract}
With the continuous development of society and economy, the development of science and technology has far exceeded the phenomenon of people. It has brought a lot of convenience and help for people's daily production and life. The development of BIM technology provides a lot of convenience for the production of the construction, and attracts the attention and research of many scholars. This paper takes BIM technology as the main research object, through the analysis and research of BIM technology optimization in project management role and its economic benefits, so as to explore the validity of the use of BIM technology, and continuously improve the advanced production in China.
\end{abstract}

\section{Overview of BIM Technology and Project Management}

BIM technology is the building information model or building information management, it builds up three-dimensional building model based on the information data of construction project, and simulates the real information of building information through digital simulation. This technology is not only a simple digital information integration, but also can be applied to digital information design, construction, all aspects of management. The main characteristic is the information completeness, information relevance, consistency, coordination, visualization, simulation, and graph optimization.

Released in May 2011, the Ministry of housing and urban development program $<<2011-2015$ construction industry information $>>$ (hereinafter referred to as the $<<$ outline $>>$ ), referred to the building information model 9 times in the overall goal and special information technology (BIM), and clearly pointed out: "12th Five-Year "period, the basic realization of information system of construction enterprise to speed up the popularization and application of BIM. The application of new technology, collaborative work in engineering based on network, promote the construction of information standard, promote with independent intellectual property rights of software industry, the formation of a number of construction enterprises in the application of information technology has reached the international advanced level; exploration and research in the design stage the 3D design technology based on BIM technology, to improve the parameterization, visualization and performance design ability, and provide technical support for the integration of design and construction; carry out the research and application of BIM technology in the construction stage, to promote BIM to extend the application of the construction stage from the design stage, the decrease in the information transmission process in the construction stage; 4D project management information system based on BIM technology in the construction process of large and complex engineering application, realize the visualization of the effective management of construction engineering. It can be said that the promulgation of the <<outline $>>$ kicked off the BIM technology in the various stages of the project management in our country and promote.

Project management is mainly refers to the project activities in the use of specialized knowledge, skills, tools and methods to make the project in the limited conditions of limited resources and achieve or exceed the set of needs and expectations. It mainly includes planning, scheduling and maintenance activities of the components in the content, and is divided into the information of project management, project management, project management, and has certain characteristics, 
namely universality, purpose, uniqueness, integration, innovation, several temporary and so on.

The project team (integration) can be defined as: "with their own objectives, combined with different professional or organization needs and culture, mutual support become consistent with the goal of the whole organization, the organization establishment of collaborative culture, highly collaborative devoted to the whole process of the project. In the construction industry, usually refers to the collaborative construction process, construction method and way of working together to create a collaborative environment all freedom of information sharing, information sharing and professional knowledge of the parties under this environment, breaking each stage of information isolation the traditional procurement model, to achieve a unified design and construction stage, improve procurement efficiency.

Project planning and management of the feasibility study stage of the management, in the stage of economic benefit and social benefit of the project, to investigate the market situation, the project feasibility analysis on the investigation results of pre construction based on the decision of the corresponding project. The content of the work is the idea of the project plan, feasibility study, etc. the maturity model structure and characteristics expressed as: in the initial stage, the owners in previous work without using BIM or BIM technology, can only use simple, rough management, BIM technology has no feasibility study to provide effective help. Growth stage order Use of BIM technology has improved, forming informal BIM technology, BIM technology in the feasibility study has been carefully conceived, the management of barely satisfactory. To improve the stage of a formal study of the use of BIM technology in the early stage in the process of management, provides support for the feasibility study, basic satisfaction the use of the results. The mature stage, use the BIM technology process has the advantages of novel design, accurate positioning, can provide information with comprehensive support for feasibility study. Continuous improvement stage, the owners of industrial BIM technology using ten skilled, can be achieved in the subsequent project continued to the use of BIM technology and constantly optimize the usage.

\section{The Optimization Function of BIM Technology in Project Management}

First of all, in the past project construction is due to measurement and management of the engineering construction of man, which appeared a lot of waste. BIM technology in the specific project management can be all construction engineering data statistics and calculation of the system, and put all the material and human needs to be applied to the project, material planning and distribution in detail, which make detailed planning and distribution, so that it can better adapt to all normal engineering construction in the process of engineering construction, so as to reduce the the traditional project for waste materials and manpower in, thus reducing the overall project expenditure. Secondly, in the control without accurate data, due to the weather, the impact of material transportation and other aspects in the implementation process of the construction of the time lag phenomenon often occurs, which need measure the overall situation in the construction of data to a certain stage, thus affecting the entire construction schedule, and the application of BIM technology can develop a complete construction plan, so as to reduce the time lag of the emergence, and guarantee the normal time.

In the current engineering construction, it has always been a time consuming and energy engineering, it relates to the reference data and the many design ideas, and the whole process of construction needs manpower to complete, which will inevitably lead to a variety of errors, such as the overall data deviation, design errors and other issues. In the conventional production buildings, these problems are often found in the whole construction process to the late, and affect the progress and quality of the entire project very much. But it can greatly improve the problem when BIM technology is applied to project management, BIM technology can integrate all the data in the early stage of engineering construction, and form a perfect construction plan, so as to monitor the parts in real time, when problems occur in the project, it will form a feedback information timely, ensure the project can be found in the early time.

BIM technology will be applied to the project management in the whole process of the project which can directly improve the quality control ability, the ability to control this is mainly 
manifested in two aspects: first, it can monitor the progress and completion of the entire project, the person in charge may arrive at the construction site without time and effort to conduct overall inspection and inquiry, the BIM technology and the system can be directly observed with the progress of the project, which can to some extent improve the whole project completion efficiency; second, at the time of the management of the project, the BIM technology can detect in the management of the project time schedule of the project, and the existing project can be timely screening and optimization, it will timely feedback the problems, and put forward some optimization programs and measures, thus to a certain extent, ensuring the quality of the entire project and facilitating the management of the entire project.

After the end of the project are summarized, so as to be able to carry out the whole life cycle of BIM application and project combination under the guidance of a round of work, from project planning to project completion, the BIM not only provides engineering modeling, hardware and other hardware support, but also regulates the modeling principle, standard. In addition, the application of BIM visualization, collaborative simulation., and figure out the characteristics of optimization, can change to the project organization, formulate relevant supporting system based on BIM environment. BIM quality control procedures and risk prevention measures in the workflow project cycle can be divided into three stages. The planning stage, this stage Based on the traditional project proposal and feasibility study report on the BIM application, develop application project of BIM target, determine the application of BIM, and the overall planning application process of BIM. The organization stage, in the application of the construction project of BIM, the application and process set after, according to the BIM of the parties involved the information coordination requirements, determine the parties involved in the BIM and the formation of the implementation team, determine the responsibilities of the parties, the parties to develop collaborative process. The implementation stage, stage and personnel involved in the division of responsibilities according to the project, the establishment of BIM application and implementation process, including the adjustment stage and the exchange of information. The support phase, at this stage, BIM applications include BIM modeling principles, standards, organizational change, quality control procedures, risk prevention measures, modeling software, hardware and so on.

Generally speaking, the BIM technology can make the whole project in the design, construction and operation phases can effectively achieve the establishment of resource planning, control financial risk, energy saving, cost saving, reduce pollution and improve the efficiency. The application of BIM technology, can change the traditional concept of project management, leading the construction of information technology to high level, thus greatly improve the degree of integration of construction management.

\section{The Economic Benefits of BIM Technology in Project Management}

BIM technology, as a high-tech technology, requires investment in technology and manpower, and the whole operation of BIM technology shows a more slow state, therefore, the economic benefits of BIM technology in project management show a lower and higher status, not only in the early economic benefit is low, but with the progress of the project continues to promote economic benefits also gradually increase.

In the process of project management, there are many related challenges we need to deal with, such as project managers cannot get effective management information needed; project managers can not get the exact prediction, data integrity; management goal of the inconsistent understanding, this is the use of maturity model value. Good project management maturity model can progress to the project management organization to provide strategic plan. Project management maturity model as a concept, provides a framework for the evaluation and improvement of enterprise project management level. The traditional project management maturity model of the project management level from a disordered state to standardized management again. To improve the optimization state is divided into five stages. The project management gradually increase the state of each stage is to achieve a higher level of a continuous improvement process of enterprise project management maturity stage is the project management level gradually improve, management experience is 
accumulated. Through the process of project management maturity model, enterprise can find out the defects in project management and identify the weak links of the project management, the project management level is crucial to solve problems form a scientific and reasonable strategies to improve project management, and steadily improve the level of enterprise project management, enterprise project management capabilities to achieve sustained improve.

To apply BIM technology to project management, the first thing we need is to purchase a large number of technical equipment, and also equipped with appropriate talent for use, which will cost a lot of investment. At the same time, in the process of using, because of the initial use, there is a certain period of running in, often there will be statistical or wrong operation problems, and need to restart the operation, in the process on time and money are wasted, which will not be related to return immediately after everything right, and even negative earnings, so early in the opinion, the overall economic benefits is low. In addition, another factor in the low economic efficiency is the BIM technology in the process of cognition is not in place, its use is not considered in all aspects of data and engineering design and using a more fixed way to set blindly, as a result, the overall utilization rate of BIM technology is low, so that the economic benefits of BIM technology cannot be maximized.

When the whole project is in its late stages, the BIM technology has reached the level of proficiency and can clearly and timely find problems in the engineering process and digest it, and manage the overall project in accordance with the instructions and functions of the system. The person in charge does not need to go out to inspect the construction site frequently and early monitoring will make the late progress of the project in accordance with the design of the original, so that reduce the waste of material and time, and the overall economic efficiency is higher. To a certain extent, the higher economic benefits are not only financial benefits, but also time benefits, at the same time, the economic benefits are not a direct economic growth, but a kind of original cost reduction, which is a form of economic growth.

BIM technology to construction project management brings convenience, BIM's goal is to work together to the construction of the whole life cycle process, BIM is the core of the construction project of information sharing and conversion, BIM is based on data standards, China is vigorously promoting the research of BIM standard, the preparation of BIM national standard is good for our country the cause of BIM from disorder to the development of orderly development, the spread in the construction industry within the scope of the whole industrial chain, healthy, it will not only lay the foundation for the construction industry to build the brand Chinese, also for the construction of green energy saving, beautiful Chinese gather strength of low carbon environmental protection technology of BIM. There, the management mode of thinking, the process was changed, which brings to the conventional management mode is a revolution. In project management, BIM is a new management mode and new management process, and is the beginning of the construction industry of fine management. Through the BIM technology as a management tool so, in the construction project managers can get more time to carry out management work, not to repeat the work, focus on the management. As the progress control work, more energy is the analysis on the rationality of schedule, rather than schedule preparation and adjustment work.

\section{References}

[1] Zhang Jianping, Liang Xiong, Liu Qiang, Wang Xiuchang, Wang Yangli, Based on BIM project management system and its application, [J]. Information technology in Civil Engineering. 2012(04)

[2] Liu Zhansheng, Zhao Ming, Xu Ruilong, Wang Zeqiang. Research and application of BIM technology in China[N]. Architectural times.2013 (04)

[3] Li Mingrui. Research on integrated management model of construction project based on BIM Technology[D]. Nanjing Forestry University 2015 
[4] Gan Lu. Study on the application of BIM technology in construction project management.[D]. Dalian University of Technology 2014

[5] Zhao Bin, Wang Youqun, Niu Bosheng. Application of 4D virtual construction technology in project schedule management based on BIM [J]. Construction economy.2011(09)

[6] Zhao Lingmin. Study on the life cycle of construction project management based on BIM [D]. shandong jianzhu university 2014

[7] Wang Bin. Research on Application of BIM technology in cost control of engineering project[D]. qingdao technological university 2015 